\title{
Protocol
}

\section{The prognostic role of immunohistochemical markers in surgical margins of Indian patients with oral squamous cell carcinoma- a retrospective study protocol}

\author{
Rajul K. Ranka*, Minal S. Chaudhary, Preethi N. Sharma, Madhuri N. Gawande
}

Department of Oral Pathology and Microbiology, Sharad Pawar Dental College and Hospital, Sawangi (Meghe), Wardha, Maharashtra, India

Received: 08 December 2020

Accepted: 23 December 2020

\author{
*Correspondence: \\ Dr. Rajul K. Ranka, \\ E-mail: rajul.ranka@gmail.com
}

Copyright: () the author(s), publisher and licensee Medip Academy. This is an open-access article distributed under the terms of the Creative Commons Attribution Non-Commercial License, which permits unrestricted non-commercial use, distribution, and reproduction in any medium, provided the original work is properly cited.

\begin{abstract}
Background: Positive surgical margins in oral squamous cell carcinoma (OSCC) is an important predictor of recurrence. Unfortunately, recurrence is found in cases with negative surgical margins as well and hence molecular markers are being investigated to identify the tumor cells in negative surgical margins to improve the survival of patients. The study aimed to ascertain the prognostic role of p53, eIF4E and E-cadherin in negative surgical margins and association of their expression with clinical parameters, recurrence, and survival.

Methods: India based retrospective cohort study of patients who were surgically treated for OSCC between 2011 and 2014. Thirty patients with negative surgical margins were assessed for this study. Sixty-four negative surgical margins were evaluated using immunohistochemistry with monoclonal mouse p53, monoclonal rabbit eIF4E and monoclonal mouse E-cadherin antibodies.

Results: Fisher's exact test, Fishers Freeman Halton exact test and contingency tables were used to assess the association between recurrence rate and clinicopathologic parameters also any association with the expression of p53, eIF4E and E-cadherin in the surgical margins and each patients' clinical characteristics. ANOVA (analysis of variance) was used to assess the role of clinicopathologic parameters in survival of the patients. The sensitivity and specificity of each antibody along with positive and negative predictive values were calculated. Kaplan Meier was used for analysis of recurrence and five-year survival. $\mathrm{P}<0.05$ was used to denote a statistically significant difference. Conclusion: This study could help in identification of patients with minimal residual cancer in the surgical margins and might help in better therapeutic management to improve survival of OSCC patients.
\end{abstract}

Keywords: E-cadherin, eIF4E, P53, Recurrence, Survival

\section{INTRODUCTION}

Oral squamous cell carcinoma (OSCC) remains the most prevalent cancer in head and neck region with its incidence increasing worldwide. In India, the maximum incidence of oral carcinoma is found in the central region. ${ }^{1}$ The 5 -year survival rate of OSCC remains low despite numerous advances in prevention and treatments like chemotherapy, radiotherapy, and targeted therapies. ${ }^{2}$ The surgical resection remains the preferred and prime treatment modality for patients with OSCC regardless of the new advancements. ${ }^{3}$ The pathology in the surgical margins have influence over survival rate of patients with OSCC as positive surgical margins increases the risk of local recurrence and poor survival of patients. ${ }^{4,5}$ But achieving a cancer clear margin at the primary location of tumor is still a challenge for most surgeons due to tumor biology and anatomic restrictions in the oral cavity. ${ }^{3}$

A clear margin is mostly cited as the cutoff distance of 5 $\mathrm{mm}$ or more of uninvolved tissue surrounding the tumor mass. ${ }^{6}$ The surgical margins are histologically evaluated 
for presence of tumor cells to check whether adjuvant treatment like chemo/radiotherapy is required by patient since tumor cells found in the margin possess risk of local recurrence. However local recurrence is often found in OSCC cases despite of histologically negative surgical margins which could be due to small number of remaining tumor cells that went undetected in routine histopathology, attributed as MRC-minimal residual cancer. $^{7}$

Immunohistochemical evaluation of negative surgical margins could embody a more reliable method to detect the MRC in them as genetic alteration goes ahead of the phenotypic changes in the epithelium. ${ }^{8}$ To establish the dependable immunohistochemical marker, the IHC marker ought to be precise for tumor cells that could distinguish between normal cells and tumor cells. ${ }^{9}$ Recent studies are centered on finding the IHC marker that may provide useful prognostic information and help in better clinical management of OSCC patients. The regular IHC evaluation of negative margins might be a better therapeutic approach over routine histopathology in recognizing patients with higher probability of local recurrence and can improve survival of OSCC patients.

p53 is a characteristic tumor suppressor protein that associates with the tumorigenesis of OSCC. It has a proven capability as a tumor marker in the evaluation of surgical margins for OSCC cases. ${ }^{10}$ p53 mutations in negative surgical margins have been shown to be valuable in predicting local recurrences in head and neck squamous cell carcinoma. ${ }^{11-13}$

Eukaryotic initiation factor 4E (eIF4E) have a crucial role in control of initial stage protein synthesis. ${ }^{14}$ It is the product of a proto-oncogene and carries immense potential as a tumor marker as it has shown overexpression in several epithelial cell tumors and has been reported to be almost $100 \%$ overexpressed in HNSCC, breast carcinomas and colon carcinomas. ${ }^{15,16}$ Few retrospective studies have shown that expression of eIF4E at negative surgical margins of HNSCC is useful in predicting local relapse..$^{13,17}$

E-cadherin is important for development and maintenance of epithelium and is one of the most essential molecules in cell-to-cell adhesion. ${ }^{18}$ The deficit of E-cadherin-mediated cell adhesion associates with the loss of the epithelial morphology and results in invasion of tumor cells. ${ }^{19}$ It has been studied in many malignant tumors as a prognostic marker and a study has reported its usefulness in the histopathological clear margins of OSCC. ${ }^{20}$

Till date, very few immunohistochemical studies has been done on surgical margins of OSCC patients in Indian population. There is a dire need to detect MRC in surgical margins to lower the incidence of recurrence, to downstage the necessity for radiotherapy, and indeed benefit the diminution of the mortality rate in OSCC patients. The present study is an endeavor to fill this research gap by utilizing the tumor markers p53, eIF4E and E-cadherin in the evaluation of histologically negative surgical margins of OSCC.

\section{Research hypothesis}

The absence of overexpression of p53 and eIF4E and presence of overexpression of E-cadherin in histopathologically negative surgical margins, individually and collectively may be an indicator of 5year survival in patients with OSCC and might be advantageous in terms of reduced recurrence and avoiding unrequired therapies.

\section{Objectives of the study}

Objectives were to evaluate the expression of p53, eIF4E and E-cadherin in histopathologically negative surgical margin of OSCC patients, to correlate the expression of p53, eIF4E and E-cadherin with postoperative 5-year survival rate of patients with OSCC and to determine the cut off values of p53, eIF4E and E-cadherin in recurrence and survival of oral squamous cell carcinoma.

\section{METHODS}

\section{Study design}

This is a retrospective cohort study carried out in the Department of Oral Pathology and Microbiology, Sharad Pawar Dental College and Hospital, India. The approval has been taken from institutional ethics committee (Ref. No. DMIMS(DU)/IEC/2017-18/7079) before the commencement of the study. The study protocol has been registered in Research Registry with a registration number research registry 6044 . We selected patients from 2011 to 2014 who were diagnosed and surgically treated at our oral cancer center. The study layout is presented in chart (Figure 1). Paraffin embedded tissue blocks of patients with histologically negative surgical margins were retrieved from departmental archives. IHC staining was done on negative surgical margins using antibodies for $\mathrm{p} 53$, eIF4E and E-cadherin and their expressions were evaluated both qualitatively and quantitatively.

The clinical and pathologic parameters of the patients included in this study were collected from archives and are described in Table 1. The association between clinical and pathologic parameters including demographic characteristics with the expression of p53, eIF4E and Ecadherin were evaluated. The recurrence of tumor in patients and the overall survival of patients were kept as primary and secondary endpoint, respectively.

\section{Selection criteria}

The target population were the patients who were surgically treated for OSCC from January 2011 to December 2014 Oral \& Maxillofacial Surgery 
Department of Sharad Pawar Dental College and Hospital. All the patients underwent radical neck dissection as their primary treatment. There was no direct involvement of patient in this study as the tissue samples were used from the departmental archives of the oral pathology department.
Inclusion and exclusion criteria: The patients diagnosed with oral squamous cell carcinoma who reported negative surgical margins in histopathology and the patients whose follow up data were available were incorporated in the study. A total of thirty patients were considered for assessment in this study.

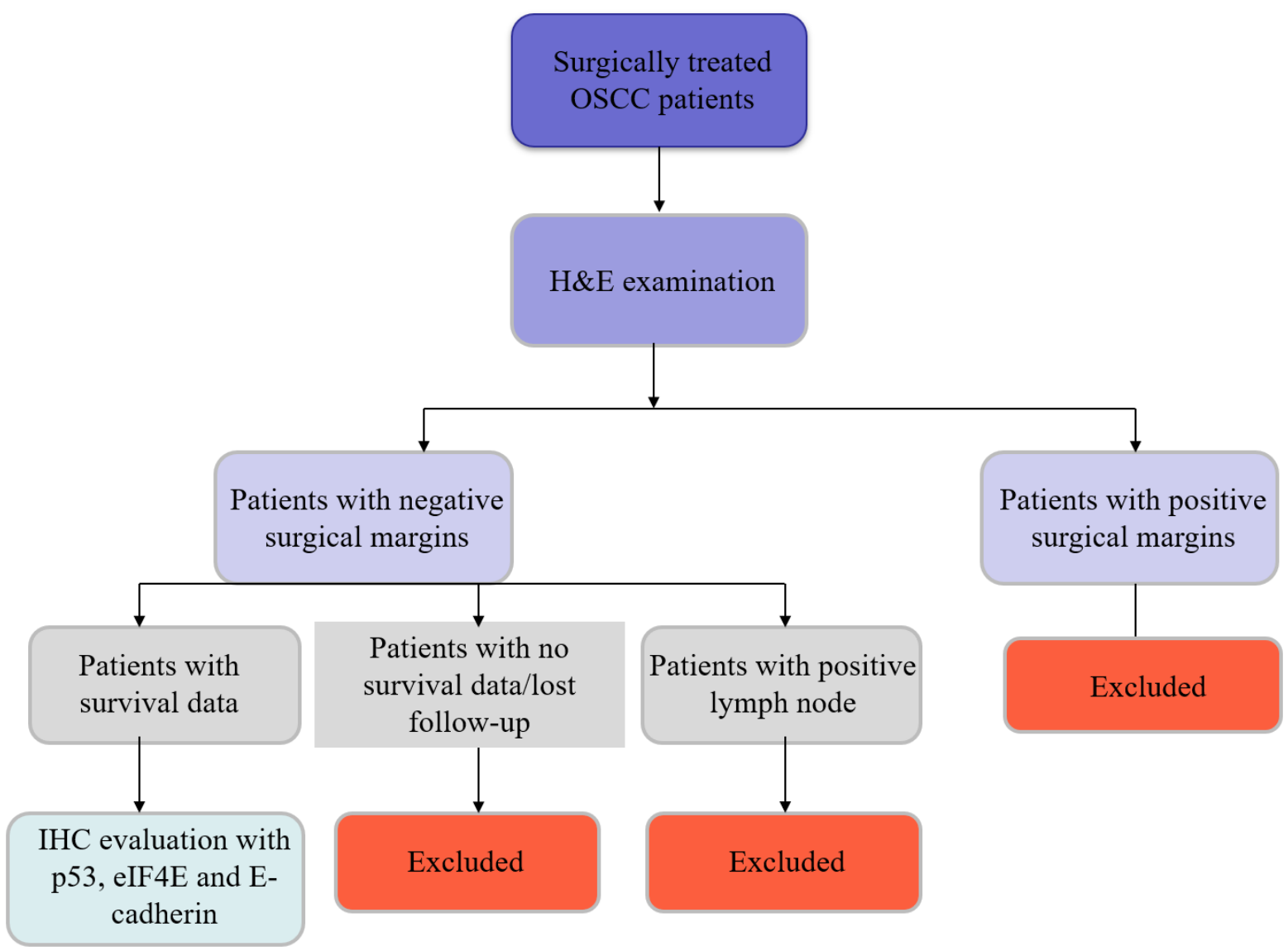

Figure 1: The study design for patient recruitment.

The patients who had positive surgical margins, had lost follow-up and who had positive lymph node metastasis in histopathology were all excluded from this study. The patients who had variants of oral squamous cell carcinoma were also excluded.

\section{Follow up period}

The patients were at follow-up starting from 6 months post-surgery until the death of the patient or 5 years. Follow-up was taken every six months by contacting patients through phone call. During the follow-up period thirteen patients had recurrence and twenty-five patients succumbed.

\section{Histopathology}

The surgical margins submitted for histopathology after surgery were included in the study. The number of margins ranged from 1 to 4 per case. A total of 64 margins were analyzed. The $\mathrm{H}$ and E-stained sections were evaluated by two investigators independently for the presence of dysplasia and the patients who had margins free of dysplasia were taken into the study.

\section{Immunohistochemistry staining}

The 3-amino propyl tri-ethoxy silane coated slides were used for section bond as well as to curtail section loss owing to pre-treatment and $4 \mu \mathrm{m}$ thin section slices were placed on them. 3 sections were taken from each surgical margin on three different slides. Slides were then labelled appropriately.

Steps in immunohistochemical staining: All the procedures were carried out following the manufacturer's instructions. The standard specific antigen retrieval was carried out for p53, eIF4E and E-cadherin using tri sodium citrate buffer $\mathrm{pH}$ for 5 minutes at $\mathrm{pH}$ 6.0. The tissue sections were hydrated with increasing grades of 
alcohol then carried to distilled water and treated with hydrogen peroxide $\left(3 \% \mathrm{H}_{2} \mathrm{O}_{2}\right)$ for 30 minutes for elimination of endogenous peroxidase activity. The tissue was sequentially incubated using Primary antibodymouse monoclonal anti-human $\mathrm{p} 53$ protein (Clone DO-7, Dako, USA) at 1:100 dilution; rabbit monoclonal recombinant anti-eIF4E antibody (Y449, Abcam, USA) at 1:250 dilution; pre-diluted mouse monoclonal Ecadherin antibody (IHC564, GeNext Genomics Pvt. Ltd, India) on room temperature in a humidifying chamber for 60 minutes followed by HRP-labeled polymer anti-mouse secondary antibody (Dako EnVision system, PC: K4000, DD.) at room temperature in a humidifying chamber for $30 \mathrm{~min}$. The newly prepared chromogen solution of DAB (3, 3' Diaminobenzidine) in the supplied buffer was used for creation of a colored precipitate at the tissue antigen sites and counterstained using Mayer's hematoxylin to visualize the antigen-antibody reaction. The slides were dehydrated using sequence of ethanol dilutions followed by xylene clearing process. The slides were then covered with cover slips by using DPX (dibutyl phthalate polystyrene xylene). Appropriate positive controls were used for p53 (oral carcinoma), eIF4E (breast carcinoma) and E-cadherin (breast carcinoma). For negative control, the primary antibody was omitted from one section in every batch. The Leica DMLB2 (Leica research microscope) at $400 \mathrm{X}$ magnification was used for evaluation of the stained sections.

\section{Microscopy}

The immunohistochemically stained surgical margins were assessed for the expression of p53, eIF4E and Ecadherin. Two investigators independently evaluated all the IHC stained slides in a blinded manner. The slides were examined both qualitatively and quantitatively. The immunostaining was considered positive for p53 antibody if dark brown staining was found in the epithelial cell nucleus of the surgical margin. The surgical margins were counted positive for eIF4E antibody if a reddishbrown peri-nuclear staining was detected in cytoplasm of the cells of the epithelial layer. E-cadherin staining was evaluated as dark brown cell membrane positivity in the epithelium of the surgical margins.

5 high power fields (HPF) with the greatest number of positive cells were evaluated per slide for p53 and eIF4E while fields with least staining of E-cadherin were selected. The staining intensity (SI) was measured as negative if no staining or weak staining in $<10 \%$ of cells, weak staining $(+)$, intermediate staining $(++)$ and strong staining $(+++)$. The percentage of positive cells (PP) were recorded as continuous variable and then were grouped as: <10\%-negative, $10-25 \%-1,26-50 \%-2,51-75 \%-3$, $\geq 75 \%-4$ for p53 and eIF4E. The percentage positive cells in E-cadherin were evaluated as: $<10 \%$ - negative, $>10 \%$ weak undetectable staining-1, $10-50 \%$ detectable discontinuous staining-2, $>50 \%$-moderate positive staining-3, >50\%-intense staining creating honey comb pattern-4. Quantification was done by recording intensity reactivity score (IRS) which is calculated as SIxPP. IRS was measured based on the score as follows: 0-0 (negative), 1-3-1 (mild), 4-6-2 (moderate), 7-9-3 (strong), 10-12-4 (intense).

Table 1: Clinical and pathological parameters of the patients.

\begin{tabular}{|l|l|}
\hline Clinicopathologic & Grouping \\
parameters & $21-30$ \\
\hline \multirow{5}{*}{ Age (year) } & $31-40$ \\
\hline & $41-50$ \\
\hline $51-60$ \\
\hline Gender & -70 \\
\hline \multirow{5}{*}{ Site of OSCC } & Male \\
\hline Female \\
\hline Oral mucosa (buccal mucosa, \\
gingivobuccal sulcus)
\end{tabular}

\section{RESULTS}

The statistical package for social sciences (SPSS Version 26, IBM, USA) was used for statistical evaluations in this research. The study assessed the outcome of survival with the expression levels of p53, eIF4E and E-cadherin. The overall survival was defined as from date of surgery to death or last information recorded. ${ }^{21}$ The time of 
recurrence was recorded from the date of surgery to the first documented recurrence. ${ }^{22}$

Continuous variables were demonstrated as mean \pm standard deviation and range. Fisher's exact test and Fisher-Freeman-Halton exact test for two categories and more than two categories respectively along with contingency tables were utilized to assess the association among clinicopathologic parameters- gender, age, tumor site, TNM staging, tumor size, nodal stage, histological grade of tumor, status of recurrence and status of survival with the expression of p53, eIF4E and E-cadherin in the surgical margins. The tests were also utilized to find the association between recurrence rate and clinicopathologic characteristics of the patients. ANOVA (analysis of variance) was used to assess the role of clinicopathologic parameters in overall survival of the patients. The correlation was carried out among expression of p53, eIF4E and E-cadherin by using the Pearson correlation and Spearman's correlation coefficient test with the level of statistical correlation significance at $\mathrm{p}<0.01$ (2-tailed). The positive predictive value and negative predictive value as well as the sensitivity and specificity for recurrence of OSCC were calculated for each antibody. The diagnostic accuracy of the tumor markers used were determined as (number of true positives and number of true negatives) / number of patients. ${ }^{23}$ Kaplan Meier, the product limit method along with log rank test was used for the event time distribution for survival and recurrence in relation to clinicopathologic parameters and expression levels of p53, eIF4E and E-cadherin. $P$ values $<0.05$ implied a statistically significant difference.

\section{Significance and outcomes}

The population studied in this research was mainly from Vidarbha region of Maharashtra and central India. So, the translation and outcomes of this proposed research study will mainly impact on the cancer care of the patients from this region. The utilization of immunohistochemical markers to detect the tumor free surgical margins might improve the survival and decrease the recurrence of OSCC. Improved patient care planning can be done in future by establishing the association between the tumor markers and clinicopathologic outcomes.

As determined by the study findings, the detection of p53, eIF4E and E-cadherin might turn out to be a regular procedure in our institute.

\section{Scope and implications}

If we confirm our research hypothesis concerning the absence of p53 and eIF4E expression and presence of Ecadherin overexpression in routine surgical margin possibly will benefit the patient to avoid additional treatment, lower the chance of local recurrence and improvement in survival, we can propose following clinical implications in our clinical pathology setting: a) routine usage of IHC markers p53, eIF4E and E-cadherin in evaluation of surgical margins of all the resected
OSCC tumors; b) Quick testing will notify the surgeons if their tumor resection is sufficient, and help in supplementary excision of the margins and/or radiation therapy to confirm complete removal of primary tumor; c) Increased disease free survival with reduced recurrence and potential metastatic disease after treatment of OSCC; d) Improved overall outcomes, thorough treatment and reduced expenses to the patient and their families.

\section{DISCUSSION}

Immunohistochemical exploration of the post-operative surgical margin in OSCC could facilitate the identification of patients with tumor free margins. It might also help to prefer the suitable adjuvant treatment options for the patients. The quick analysis might help surgeons in determining the exact margins and aid in further excision of the tumor.

The combination of p53, eIF4E and E-cadherin tumor markers has not been studied. The role of these markers is significant in future management of OSCC. The study attempts to find the best and reliable marker for Indian population. These markers could be used on daily basis to detect MRC delivering patient with comprehensive management. There is a strong hope that these tumor markers will substantially contribute to the field of oral and maxillofacial surgery and pathology by aiding enhanced therapeutic management of patients in India.

\section{ACKNOWLEDGEMENTS}

Author would like to thank Sharad Pawar dental college and hospital for providing infrastructural facilities to conduct this research. Autor is also thankful to $\mathrm{Mr}$. Rupesh Maladhari, histopathology and immunohistochemistry laboratory technician for all his technical support.

Funding: The financial assistance for antibodies used in the study was provided by Datta Meghe Institute of Medical Sciences (deemed to be university).

Conflict of interest: None declared

Ethical approval: The study was approved by the Institutional Ethics Committee-Ref. No. DMIMS(DU)/IEC/2017-18/7079

\section{REFERENCES}

1. Sharma S, Satyanarayana L, Asthana S, Shivalingesh K, Goutham BS, Ramachandra S. Oral cancer statistics in India on the basis of first report of 29 population-based cancer registries. J Oral Maxillofac Pathol. 2018;22:18-26.

2. Taghavi N, Yazdi I. Prognostic factors of survival rate in oral squamous cell carcinoma: Clinical, histologic, genetic and molecular concepts. Arch Iran Med. 2015;18:314-9.

3. Stathopoulos P, Smith WP. Close Resection Margins Do Not Influence Local Recurrence in Patients with 
Oral Squamous Cell Carcinoma: A Prospective Cohort Study. J Oral Maxillofac Surg. 2018;76:8736.

4. Stathopoulos P, Smith PW. Analysis of survival rates following primary surgery of 178 consecutive patients with oral cancer in a large district general hospital. J Maxillofac Oral Surg. 2017;16:158.

5. Sutton DN, Brown JS, Rogers SN, Vaughan ED, Woolgar JA. The prognostic implications of the surgical margin in oral squamous cell carcinoma. Int J Oral Maxillofac Surg. 2003;32:30.

6. Tasche KK, Buchakjian MR, Pagedar NA, Sperry SM. Definition of "Close Margin" in Oral Cancer Surgery and Association of Margin Distance with Local Recurrence Rate. JAMA Otolaryngol Head Neck Surg. 2017;143:1166-72.

7. Braakhuis BJ, Bloemena E, Leemans CR, Brakenhoff RH. Molecular analysis of surgical margins in head and neck cancer: more than a marginal issue. Oral Oncol. 2010;46:485-91.

8. Ranka R, Chaudhary M, Sharma P. Role of Immunohistochemical Markers in Surgical Margins of Patients with Head and Neck Carcinoma-A Systematic Review. Int J Cur Res Rev. 2020;12:917.

9. Roncella S, Ferro P, Bacigalupo B, Tognoni A, Falco E, Gianquinto D et al. Human mammaglobin mRNA is a reliable molecular marker for detecting occult breast cancer cells in peripheral blood. J Exp Clin Cancer Res. 2005;24:265-71.

10. Brennan JA, Mao L, Hruban RH, Boyle JO, Eby YJ, Koch WM, et al. Molecular assessment of histopathological staging in squamous-cell carcinoma of the head and neck. $\mathrm{N}$ Engl J Med. 1995;332:429-35.

11. Van Houten VM, Leemans CR, Kummer JA, Dijkstra J, Kuik DJ, van den Brekel MW et al. Molecular diagnosis of surgical margins and local recurrence in head and neck cancer patients: a prospective study. Clin Cancer Res. 2004;10:361420.

12. Huang X, Pateromichelakis S, Hills A, Sherriff M. p53 mutations in deep tissues are more strongly associated with recurrence than mutation positive mucosal margins. Clin Cancer Res. 2017;13:6099106.

13. Singh J, Jayaraj R, Baxi S, Mileva M, Skinner J et al. Immunohistochemical expression levels of p53 and eIF4E markers in histologically negative surgical margins, and their association with the clinical outcome of patients with head and neck squamous cell carcinoma. Mol Clin Oncol. 2016;4:166-72.
14. Han M, Wang W, Wang L, Jiang Y. Expression of eukaryotic initiation factor $4 \mathrm{E}$ in hypopharyngeal carcinoma. J Int Med Res. 2014;42:976-83.

15. Culjkovic B, Borden KL. Understanding and Targeting the Eukaryotic Translation Initiation Factor eIF4E in Head and Neck Cancer. J Oncol. 2009;2009:981679.

16. Wang XL, Cai HP, Ge JH, Su XF. Detection of eukaryotic translation initiation factor $4 \mathrm{E}$ and its clinical significance in hepatocellular carcinoma. World J Gastroenterol. 2012;18:2540-4.

17. Nathan CO, Liu L, Li BD, Abreo FW. Detection of the proto-oncogene eIF4E in surgical margins may predict recurrence in head and neck cancer. Oncogene. 1997;15:579-84.

18. Bengallo T, El-Faitori M, Sassi S, Khaial FB, El Gehani K, Buhmeida A et al. Expression of ECadherin in Prostatic Carcinoma: Prognostic Significance. J Cancer Prev Curr Res. 2016;4:00119.

19. Efstathiou JA, Liu D, Wheeler JM, Beck NE, Ilyas M, Karayiannakis AJ et al. Mutated epithelial cadherin is associated with increased tumorigenicity and los of adhesion and of responsiveness to the motogenic trefoil factor 2 in colon carcinoma cells. Proc Natl Acad Sci USA. 1999;96:2316-21.

20. Mohtasham N, Anvari K, Memar B, Saghravanian N, Ghazi N, Bagherpour A et al. Expression of Ecadherin and matrix metalloproteinase-9 in oral squamous cell carcinoma and histologically negative surgical margins and association with clinicopathological parameters. Rom J Morphol Embryol. 2014;55:117-21.

21. Waitzberg AF, Nonogaki S, Nishimoto IN, Kowalski LP, Miguel RE, Brentani RR et al. Clinical significance of c-myc and p53 expression in head and neck squamous cell carcinomas. Cancer Detect Prev. 2004;28:178-86.

22. Nathan CAO, Sanders K, Abreo FW, Nassar R, Glass J. Correlation of p53 and the proto-oncogene eIF4E in larynx cancers: prognostic implications. Cancer Res. 2000;60:3599.

23. Ogbureke KU, Weinberger PM, Looney SW, Li L, Fisher LW. Expressions of matrix metalloproteinase9 (MMP-9), dentin sialophosphoprotein (DSPP), and osteopontin (OPN) at histologically negative surgical margins may predict recurrence of oral squamous cell carcinoma. Oncotarget. 2012;3:286-98.

Cite this article as: Ranka RK, Chaudhary MS, Sharma PN, Gawande MN. The prognostic role of immunohistochemical markers in surgical margins of Indian patients with oral squamous cell carcinoma-a retrospective study protocol. Int J Clin Trials 2021;8(1):58-63. 